\section{Imaging Large Tissue Samples at High Resolution}

Robert Meyer, Meyer Instrument Inc. rdm@meyerinst.com

To those scientists and researchers who use microscopes with video or digital cameras for the purpose of capture and display, there has always existed a fundamental problem, namely, imaging large tissue samples at high resolution.

For some time, conventional technology has allowed video cameras to be attached to microscopes. The signal generated by these cameras can be captured and saved to a computer's hard drive using a frame grabber. A typical image capture and display resolution is 640 by $480(307,200)$ pixels. Although the image produced is 'good', it is by no means considered 'high resolution'. For the sake of discussion, 'high resolution' is defined as greater than $1,000 \times 1,000(1,000,000)$ pixels. High resolution images are essential for medical applications. Today's modern digital cameras can meet or exceed this value. The human eye, if compared to these examples, has the equivalent resolution of greater than $5,000 \times 5,000$ $(25,000,000)$ pixels.

When viewing through a standard laboratory grade microscope with a low power objective, (i.e., $1 \mathrm{x}, 2.5 \mathrm{x}, 4 \mathrm{x}$ ), the visual field of view ranges from approximately $22 \mathrm{~mm}$ to $5.5 \mathrm{~mm}$ (research microscopes can provide ranges from $28 \mathrm{~mm}$ to 7 $\mathrm{mm}$ ). This is a circular field of view. When a video or digital camera is added to the microscope the field of view decreases to approximately $16 \mathrm{~mm}$ to $4 \mathrm{~mm}$. This is a rectangular field of view with generally a 4 by 3 aspect ratio. In other words, the largest sample a microscopist can view on a video monitor is limited to approximately $16 \mathrm{~mm}$ by $12 \mathrm{~mm}$. An average microscope video/digital capture and display system costs between $\$ 15,000.00$ and $\$ 25,000.00$. Larger fields and higher capture and display resolutions are possible with research microscopes, motorized stages, additional intermediate lenses and high performance capture and display cards, but for tens of thousands of dollars more.

Microscope glass specimen slides, by historical design, are $76.2 \mathrm{~mm}$ wide by $25.4 \mathrm{~mm}$ tall by $1.1 \mathrm{~mm}$ thick (commonly referred to as a $1 \times 3$ inch glass slide). Samples, usually histological tissue, are prepared and sliced into very thin 3 to $5 \mathrm{mi}-$ crons sections. These sections are mounted on the glass slide and protected by a coverslip with dimensions of usually $40 \mathrm{~mm}$ by $24 \mathrm{~mm}$ by $0.15 \mathrm{~mm}$. As a result, the largest tissue sample is limited by the size of the coverslip. These slides are viewed by pathologists, anatomists, biologists, etc., on an everyday routine basis. Literally millions of slides are created and viewed by thousands of researchers and clinicians every year. The need to capture and display these samples is obvious.

As stated, once the sample exceeds $16 \mathrm{~mm}$ by $12 \mathrm{~mm}$ a microscopist has a problem. Even though the slide and coverslip can accommodate a sample section of up to $40 \mathrm{~mm}$ by 24 $\mathrm{mm}$, there is no way, using a microscope and capture system, one can visualize or display the entire sample (let alone in high resolution). The PathScan Enabler solves this problem.

Over the last several years new products have emerged on the market that allows one to scan $35 \mathrm{~mm}$ photographic slides and/or negatives directly to a computer's hard drive. As an example, a device offered by the Polaroid Corporation called the SprintScan 4000 Digital Film Scanner allows an entire $36 \mathrm{~mm}$ by $24 \mathrm{~mm}$ color slide to be scanned at 4000 dots per inch (dpi) in just over one (1) minute. Most film scanners operate by moving a sample in front of a tri-linear charged couple device (CCD) array. The result is an extremely high resolution, accurate replication of the information contained in the slide.

If one were to replace a $35 \mathrm{~mm}$ slide with a microscope glass specimen slide and a holder to accommodate such slide, sample tissue could be scanned and displayed at a resolution much higher than the conventional $640 \times 480$ pixels, in fact as high as $5600 \times 4080$. The PathScan Enabler is such a holder and works exclusively with the Polaroid SprintScan 4000 Digital Film Scanner. The patented PathScan Enabler consists of an aluminum housing and a proprietary optically flat neutral density filter. It fits into a modified $35 \mathrm{~mm}$ slide carrier that in turn fits into the SprintScan 4000.

The images produces with these instruments are simply gorgeous! They are evenly illuminated, incredibly sharp and color perfect. Because there is so much detail an image produced with the PathScan Enable can be enlarged and printed to as much as 8 feet in diameter! Most importantly the researcher can see the WHOLE sample with stunning clarity and detail. The PathScan Enabler and Polaroid 4000 Digital Film scanner are perfect compliments to a video/digital microscope imaging system.

The Pathscan Enabler's retail list price is $\$ 599.00$. When purchased with a SprintScan 4000 and an appropriate computer system, the total list price is around $\$ 4,800.00$. A comparable video/digital microscope capture and display system, including computer, microscope, a $1.0 x$ objective, programmable motorized stage, frame grabber, video camera, display monitor, and capture software would total around $\$ 30,000.00$.

Which would you want to buy?

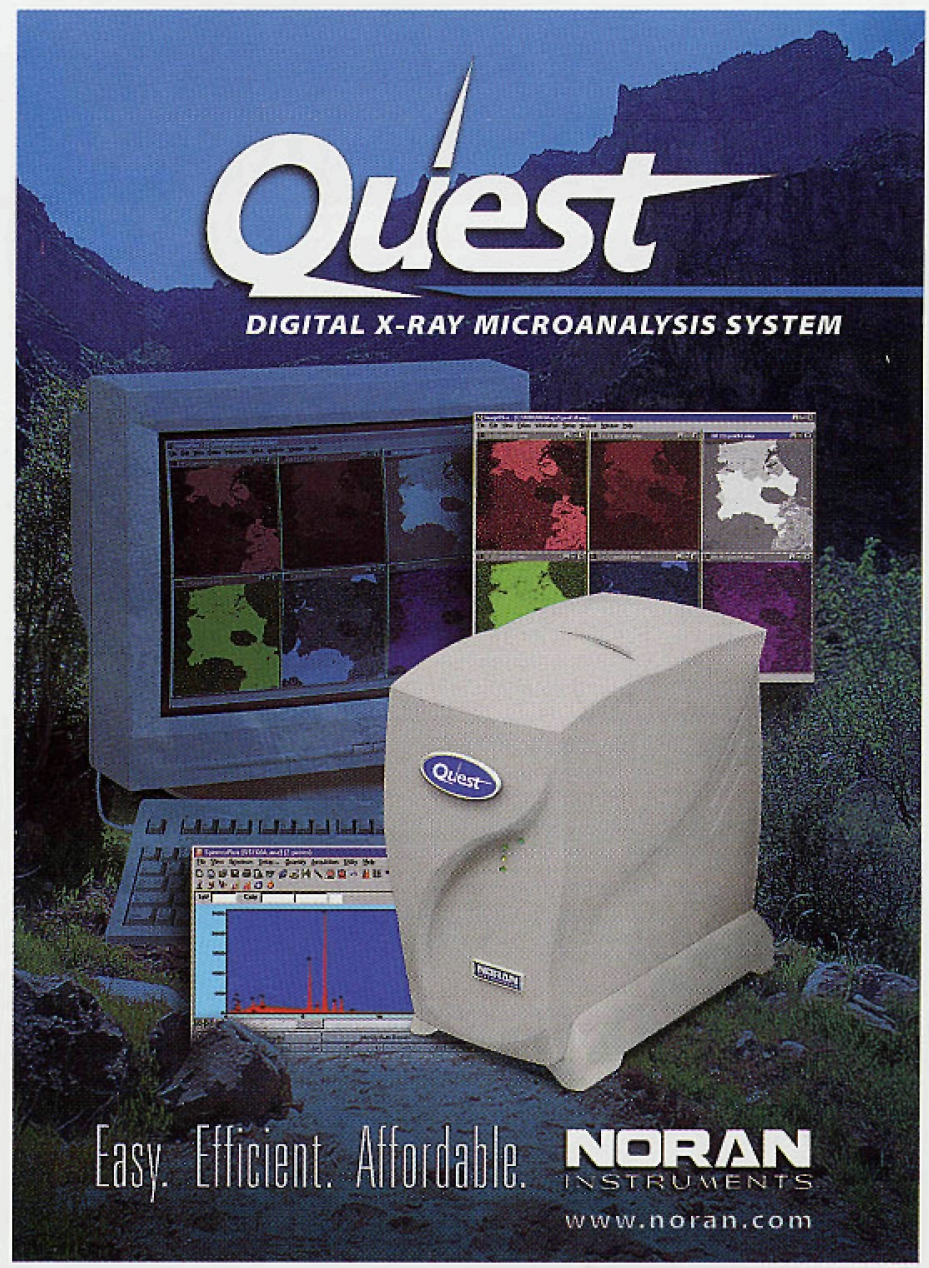

